\title{
Limestone calcination optimization of vertical container oven
}

\author{
${ }^{a}$ Alexis Sagastume Gutiérrez. \\ Universidad de Cienfuegos. \\ E-mail: asagastume@ucf.edu.cu.
}

\begin{abstract}
Producing lime demand high energy consumption and implies high $\mathrm{CO} 2$ emissions and other environmental impacts significant. This work aims to establish a methodology for optimizing the calcination of limestone kilns normal vertical Cuba, to consider the influence of the quality of lime on the economic contribution margin. In this case they are used as tools work the method of exergy analysis and the method of genetic algorithms was used. The exergy balance limestone calcination identified that irreversibilities of combustion and heat transfer time and exergy loss in the exhaust gases, are the principal process inefficiencies. The implementation of the proposed optimization methodology reduced the energy consumption by $4.6 \%$ and increased the quality of lime at $5.3 \%$.
\end{abstract}

\section{Keywords:}

\section{Optimización de la calcinación de calizas en hornos de cuba vertical}

\section{RESUMO}

La producción de cal demanda elevados consumos energéticos e implica altas emisiones de $\mathrm{CO} 2$, así como importantes otros impactos ambientales. Este trabajo está dirigido a establecer una metodología de optimización de la calcinación de calizas en hornos de cuba vertical normal, que considere la influencia de la calidad de la cal sobre el margen de contribución económica. En este caso se utilizan como herramientas el trabajo se utilizó el método de análisis exergético y el método de los Algoritmos Genéticos. El balance exergético de la calcinación de calizas, identificó que las irreversibilidades de la combustión y de la transferencia de calor y momento, así como la pérdida de exergía con los gases de escape, constituyen las principales inefíciencias del proceso. La implementación de la metodología de optimización propuesta redujo el consumo energético en un 4,6\% y aumentó la calidad de la cal en un 5,3\%.

\section{Palabras Clave:}

\section{INTRODUÇÃO}

La cal es el alcalino más barato que existe y es una materia prima vital para muchos procesos industriales [1]. El proceso de producción de la cal es un alto consumidor de energía, lo que además representa su principal costo de producción (alrededor del 50\% del costo total de producción) [2, 3]. Estas características han provocado que la crisis energética y el incremento de los precios del combustible afecten de manera particular los costos de producción de esta industria [4, 5]. Otro factor importante, también relacionado con el consumo energético, es la emisión de $\mathrm{CO} 2$, principal factor del cambio climático [6]. En la producción de una tonelada de cal viva se emiten alrededor de 1,2 t de $\mathrm{CO} 2$ [7,8]; siendo el proceso de calcinación el proceso más contaminante de la producción de cal [9]. El consumo de energía además influye de forma directa sobre la calidad de la cal viva $[1,10,11,12,13]$ que depende de su contenido de $\mathrm{CaO}$.

La calcinación de calizas se desarrolla en hornos diseñados con ese objetivo, de los cuales existen diferentes diseños que se clasifican en tres grandes grupos [14]: hornos artesanales, hornos de cuba vertical y hornos rotatorios. Siendo los hornos de cuba vertical los de mayor eficiencia térmica $[1,2$, 15]. En las plantas existentes en Cuba se explotan de forma exclusiva hornos de cuba vertical normal.

Un horno de cuba vertical normal (ver Fig. 1), básicamente consiste en un reactor de lecho móvil, en el cual los gases calientes ascienden a contracorriente con las rocas calizas, que descienden mientras se calcinan a elevadas temperaturas, normalmente por encima de los $900{ }^{\circ} \mathrm{C}$. Para su análisis, el horno 
Alexis Sagastume Gutiérrez / ITEGAM-JETIA Vol.01, No 01, pp. 11-18. Março, 2015.

se divide en tres partes: zona de precalentamiento, zona de calcinación y zona de enfriamiento. La zona de calcinación comienza en el punto donde se inicia la disociación de los carbonatos de calcio en la roca y la zona de enfriamiento comienza en el punto donde cesa la calcinación. Las rocas calizas (y el combustible sólido en caso de un horno de mezcla) se alimentan por la parte superior del horno y la cal viva producida es descargada por la parte inferior del mismo. Los quemadores de gas o de petróleo se ubican en la zona de calcinación. La localización de estas zonas es una definición convencional y no física; por otra parte, su altura es variable y depende de las condiciones de operación del horno.

El calor requerido para la disociación de la roca se suministra mediante la combustión del combustible apropiado (carbón, petróleo, gas, etc.).

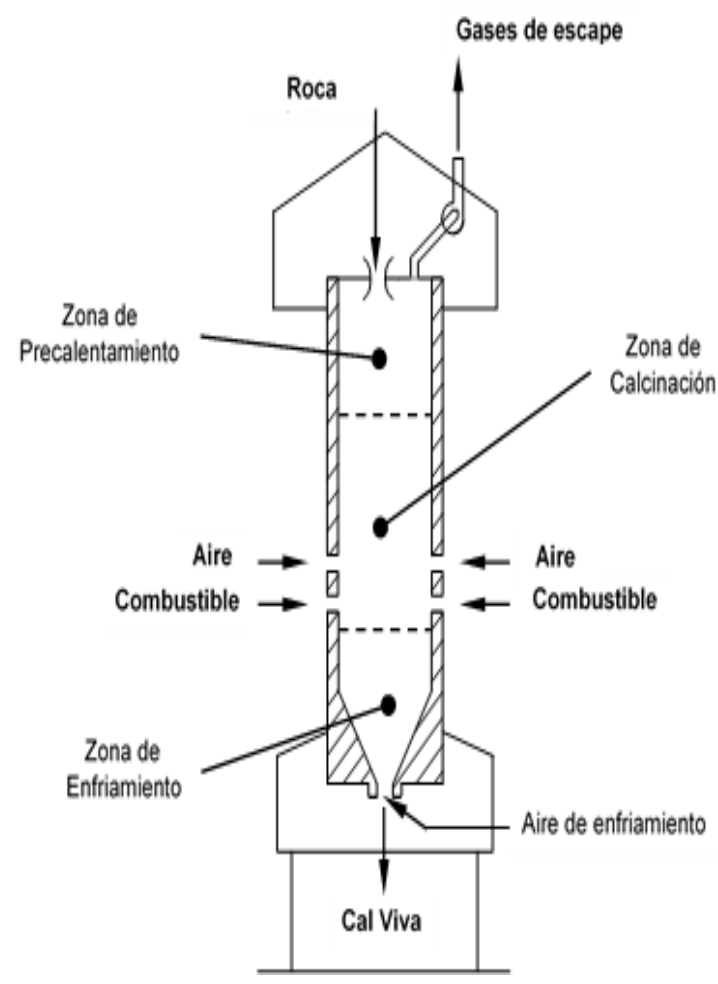

Figura 1. Esquema de un horno de cuba vertical normal.

A pesar de esto, se reportan pocas investigaciones en la literatura especializada sobre la evaluación y/o optimización del consumo de energía en este proceso [3, 14, 16, 17, 18]. En general, las propuestas descritas por la literatura se fundamentan en el 1er principio de la termodinámica $[14,16,17,18,19,20]$, implican la introducción de nuevas tecnologías en el proceso [21, 22, 23] o el uso de personal altamente capacitado [24] u optimizan desde el diseño [25, 26, 27, 28, 29]. Los enfoques del 1er principio de la termodinámica no permiten identificar todas las pérdidas del proceso, ni todas las potencialidades para reducir el consumo de combustible; el resto de los enfoques implican inversiones importantes que muchas veces no están al alcance de pequeñas plantas de cal. En ningún caso se evalúa la dimensión ambiental de la producción de cal ni se tiene en cuenta la influencia de la calidad de la cal sobre su precio de venta.

El objetivo de este trabajo es presentar una metodología de optimización de la calcinación de calizas en hornos de cuba vertical que considere la influencia de la calidad de la cal sobre su precio de venta.

\section{METODOLOGÍA}

Los principales objetivos cuando se optimiza el funcionamiento de un horno de producción de cal son [30]: reducir el consumo de combustible, acelerar el proceso de producción y mejorar el aprovechamiento de la materia prima (roca caliza). Estos objetivos, están influenciados por los parámetros de operación del horno, que están vinculados a sus variables de control:

- $\quad$ Flujo de alimentación combustible (F)

- Flujo de alimentación de roca caliza (Ls)

- $\quad$ Flujo de extracción de cal viva (Q1)

- $\quad$ Coeficiente de exceso de aire $(\lambda)$

- $\quad$ Tiro del horno $(\Delta \mathrm{P})$

- $\quad$ Radio medio de las rocas $(\mathrm{R})$

- $\quad$ Porosidad del lecho $(\psi)$

- Masa de aire de enfriamiento $\left(\mathrm{ma}_{\mathrm{S}}\right)$

El objetivo de la optimización definir el valor de las variables de control que optimizan el margen de contribución económica $(\mathrm{G})$ del proceso, entendido como la diferencia entre el precio de venta de la cal $(\mathrm{P})$ y su costo de producción $\left(\mathrm{C}_{\mathrm{P}}\right)$ :

$$
\max \mathrm{G}=\mathrm{P}-\mathrm{C}_{\mathrm{p}}
$$

La calidad de la cal es función del porciento de $\mathrm{CaO}$ $\left(\mathrm{A}_{\mathrm{q}}\right)$ (que define el grado de conversión $(\mathrm{X})$ de la calcinación), en el mercado mundial se cotiza aproximadamente según muestra la tabla 1 .

Tabla 1. Precio de la cal en función de $\mathrm{A}_{\mathrm{q}}$.

\begin{tabular}{|c|c|}
\hline $\mathbf{A}_{\mathbf{q}}(\boldsymbol{\%})$ & $\mathbf{P}(\mathbf{\$} / \mathbf{t})$ \\
\hline 75 & 40 \\
\hline 80 & 60 \\
\hline 85 & 88 \\
\hline 90 & 110 \\
\hline 95 & 128 \\
\hline 100 & 132 \\
\hline
\end{tabular}

De donde se define una ecuación para calcular el precio de la cal en función de su calidad: 
Alexis Sagastume Gutiérrez / ITEGAM-JETIA Vol.01, No 01, pp. 11-18. Março, 2015.

$$
P=132,35 \cdot \exp \left[\frac{-\left(0,9928-A_{q}\right)^{2}}{2 \cdot 0,1556^{2}}\right]
$$

El costo de producción de la cal viva es función de los costos variables y los costos fijos. En este caso se considera el costo de la roca alimentada al horno $\left(\mathrm{C}_{\mathrm{R}}\right)$, el costo de la electricidad utilizada $\left(\mathrm{C}_{\mathrm{E}}\right)$, el costo de la mano de obra $\left(\mathrm{C}_{\mathrm{M}}\right)$, el costo del combustible consumido por el proceso $\left(\mathrm{C}_{\mathrm{F}}\right)$, la amortización $\left(\mathrm{C}_{\mathrm{A}}\right)$, el impuesto de la fuerza de trabajo $\left(\mathrm{C}_{\mathrm{FT}}\right)$, el costo por reparación y mantenimientos $\left(\mathrm{C}_{\mathrm{RM}}\right)$, el gasto general de administración $\left(\mathrm{C}_{\mathrm{Adm}}\right)$ y otros gastos $\left(\mathrm{C}_{\mathrm{O}}\right)$ :

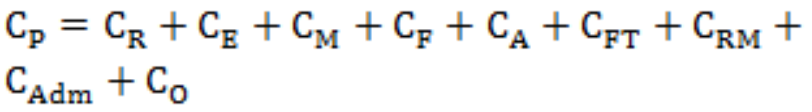

Los costos variables de producción son una función directa de la eficiencia en el consumo de energía y materias primas que dependen del consumo de roca caliza, electricidad y fuel, siendo estos los factores más influyentes en el desempeño ambiental de la producción de cal [9]. Por consiguiente, al optimizar la función objetivo, no solo se optimiza el desempeño económico de la calcinación; sino que además se optimiza su desempeño energético y ambiental.

El primer paso de la metodología propuesta (ver fig. 2) es definir los costos de operación del horno. Posteriormente se caracteriza el estado de operación del horno, es decir, se miden los parámetros de control del horno. Una vez caracterizado el horno, se realiza el balance exergético para identificar las ineficiencias del proceso, y la influencia de cada parámetro de operación sobre cada una de ellas. Mediante este análisis, se identifican los parámetros de operación que más influyen sobre la ineficiencia del horno. Una vez identificados los parámetros a optimizar, se implementa el método de los Algoritmos Genéticos para optimizar. Finalmente se implementan los resultados en el horno.

El balance de exergía del horno se desarrolló según se describe en [31]. El modelo matemático utilizado se describe en $[2,32,33]$. La optimización se desarrolla utilizando el método de los algoritmos genéticos [34].

\section{RESULTADOS Y DISCUSIÓN}

Para implementar la metodología se seleccionó un horno de cuba vertical en una planta de cal Cuba. El horno analizado produce un promedio de 45 toneladas de cal viva diaria y utiliza fueloil como combustible, con un coeficiente de exceso de aire que alcanza valores de 1,9. Para establecer una línea de comparación se desarrolla el balance para un horno de alta eficiencia en la planta suiza Kalkfabric Nestal (KFN) reportado en la literatura [35] con una producción diaria de 50,6 toneladas $y$ un coeficiente de exceso de aire de 1,05.
Para implementar la metodología propuesta se definieron los costos de operación del horno (ver tabla 2).

Tabla 2. Costos de producción de la cal viva.

\begin{tabular}{|c|c|c|}
\hline Concepto & Unidad & $\begin{array}{c}\text { Costo } \\
\text { (\$/Unidad) }\end{array}$ \\
\hline Combustible & $\mathrm{l}$ & 0,83 \\
\hline Electricidad & $\mathrm{kWh}$ & 0,23 \\
\hline $\begin{array}{c}\text { Roca alimentada al } \\
\text { horno }\end{array}$ & $\mathrm{t}$ & 8,46 \\
\hline Mano de obra & $\mathrm{t}$ & 6,31 \\
\hline Amortización & $\mathrm{t}$ & 0,34 \\
\hline $\begin{array}{c}\text { Impuesto de la } \\
\text { fuerza de trabajo }\end{array}$ & $\mathrm{t}$ & 1,38 \\
\hline $\begin{array}{c}\text { Reparación y } \\
\text { mantenimiento }\end{array}$ & $\mathrm{t}$ & 1,66 \\
\hline $\begin{array}{c}\text { Gasto de } \\
\text { administración }\end{array}$ & $\mathrm{t}$ & 0,01 \\
\hline Otros gastos & $\mathrm{t}$ & 0,07 \\
\hline
\end{tabular}


Alexis Sagastume Gutiérrez / ITEGAM-JETIA Vol.01, No 01, pp. 11-18. Março, 2015.

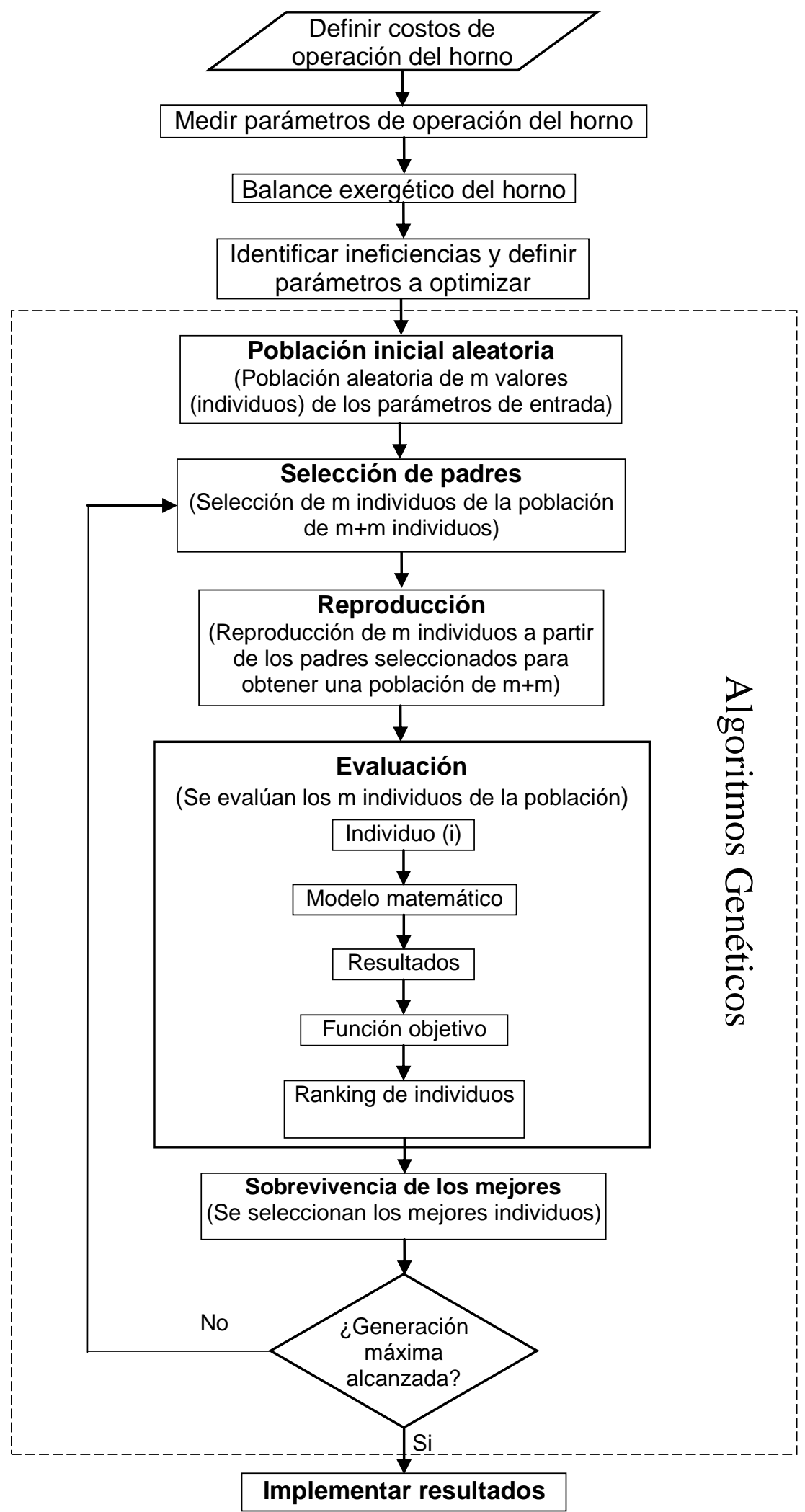

Figura 2. Metodología de optimización 
Como segundo paso de la metodología de optimización, se miden los parámetros de operación del horno durante tres días. La tabla 3 muestra los resultados de la medición y de la eficiencia exergética, el precio de la cal, los costos de producción y el margen de contribución económica, calculados a partir de las mediciones. Como se puede apreciar en la tabla, para las condiciones de explotación actual, la planta opera con pérdidas económicas. Esto se debe en lo fundamental a la mala operación del opera que provoca una baja eficiencia exergética con un consumo de combustible alto.

Para identificar las ineficiencias asociadas a la calcinación de calizas, se desarrolla el balance exergético como se muestra en la fig. 3. Además se analiza el horno de KFN.

Tabla 3. Parámetros de operación medidos en el horno.

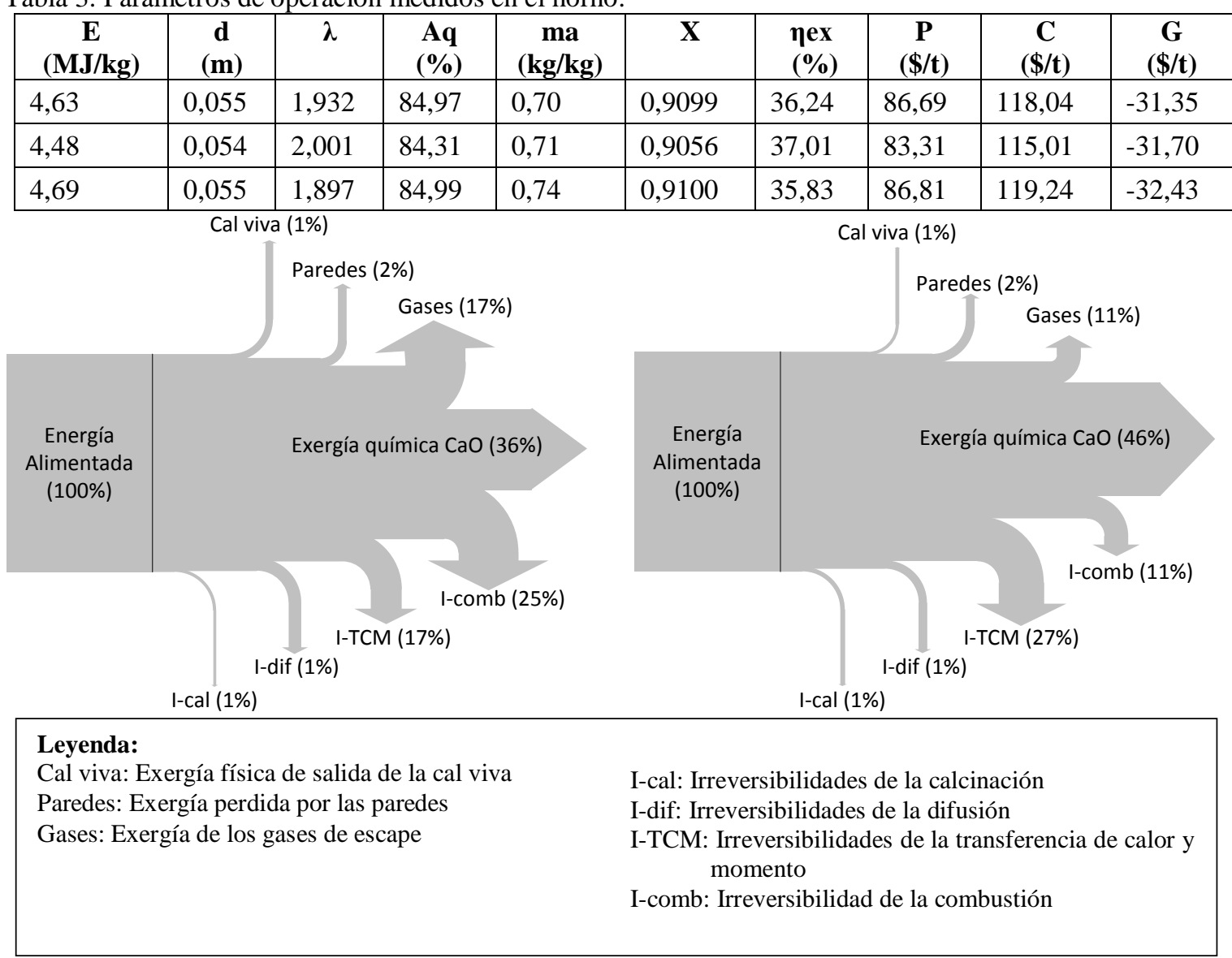

Figura 3. Balance exergético del horno.

En la figura se aprecia que en el horno estudiado las mayores ineficiencias se concentran en las irreversibilidades de la combustión, la transferencia de calor y momento y la pérdida de exergía con los gases de escape sumando el 59\% de la exergía alimentada al horno. Por otra parte, en el horno de KFN las irreversibilidades de la transferencia de calor y momento constituyen la principal pérdida de eficiencia y sumado a las irreversibilidades de la combustión y a la pérdida de exergía de los gases de escape suma el $49 \%$ de la exergía alimentada al horno perdida en el proceso.
La optimización de la operación horno está enfocada a definir el valor de los parámetros de control que maximizan el margen de contribución económica (G). En este caso, considerando las características de los hornos de cuba vertical y los datos estadísticos de la explotación de estos hornos en Cuba y el consumo mínimo posible de esta tecnología, el consumo energético estará limitado entre 4,1 y $5,5 \mathrm{MJ} / \mathrm{kg}_{\mathrm{Ql}}$. Considerando los resultados del balance exergético, se enfatiza en el control del coeficiente de exceso de aire en la operación del horno. Para la optimización se mantuvieron 
constantes el diámetro de la roca, el coeficiente de exceso de aire y la masa de aire de enfriamiento, optimizándose el consumo de combustible. Los resultados de la optimización se muestran en la tabla
4. Como último paso de la metodología se implementan los resultados en el horno. En este caso se trabajó durante tres días en la implementación los resultados se muestran en la tabla 5 .

Tabla 4. Resultados de la optimización.

\begin{tabular}{|c|c|c|c|c|c|c|c|c|c|}
\hline $\begin{array}{c}\mathbf{E} \\
(\mathbf{M J} / \mathbf{k g})\end{array}$ & $\begin{array}{c}\mathbf{d} \\
(\mathbf{m})\end{array}$ & $\boldsymbol{\lambda}$ & $\begin{array}{c}\mathbf{A q} \\
(\boldsymbol{\%})\end{array}$ & $\begin{array}{c}\mathbf{m a} \\
(\mathbf{k g} / \mathbf{k g})\end{array}$ & $\mathbf{X}$ & $\boldsymbol{\eta} \mathbf{e x}(\boldsymbol{\%})$ & $\mathbf{P}(\mathbf{\$} / \mathbf{t})$ & $\mathbf{C}(\mathbf{\$} / \mathbf{t})$ & $\mathbf{G}(\mathbf{\$} / \mathbf{t})$ \\
\hline 4,40 & 0,055 & 1,550 & 93,89 & 0,93 & 0,9845 & 42,09 & 124,62 & 114,00 & 10,62 \\
\hline
\end{tabular}

Tabla 5. Parámetros de operación medidos durante la optimización del horno.

\begin{tabular}{|c|c|c|c|c|c|c|c|c|c|}
\hline $\begin{array}{c}\mathbf{E} \\
\mathbf{M J} / \mathbf{k g})\end{array}$ & $\begin{array}{c}\mathbf{d} \\
(\mathbf{m})\end{array}$ & $\boldsymbol{\lambda}$ & $\begin{array}{c}\mathbf{A q} \\
\mathbf{( \% )}\end{array}$ & $\begin{array}{c}\mathbf{m a} \\
\mathbf{( k g} / \mathbf{k g})\end{array}$ & $\mathbf{X}$ & $\boldsymbol{\eta} \mathbf{e x}(\mathbf{\%})$ & $\mathbf{P}(\mathbf{\$} / \mathbf{t})$ & $\mathbf{C}(\mathbf{\$} / \mathbf{t})$ & $\mathbf{G}(\mathbf{\$} / \mathbf{t})$ \\
\hline 4,42 & 0,055 & 1,573 & 92,94 & 0,92 & 0,9788 & 41,49 & 121,80 & 114,61 & 7,19 \\
\hline 4,41 & 0,055 & 1,557 & 92,86 & 0,94 & 0,9783 & 41,55 & 121,53 & 114,41 & 7,13 \\
\hline 4,36 & 0,055 & 1,510 & 93,15 & 0,94 & 0,9800 & 42,14 & 122,46 & 113,43 & 9,03 \\
\hline
\end{tabular}

Comparando las tablas 4 y 5 puede observarse una mejora en el desempeño de la calcinación de calizas. También se aprecia una reducción de los costos de producción y un aumento del precio de venta del producto, incrementando el margen de contribución económica. En este caso se redujo el consumo de combustible (reduciendo los costos de producción), mejorándose además la calidad de la cal viva (aumentando el precio de venta). Con la implementación de los resultados la planta comenzó a operar con beneficios económicos.

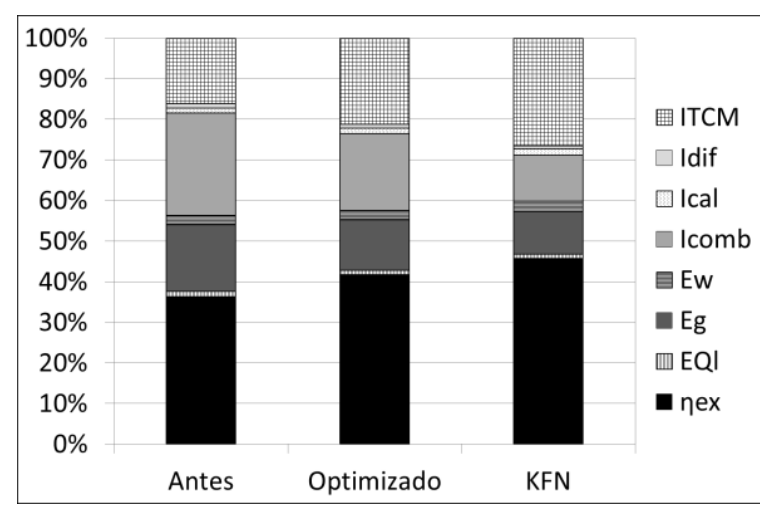

Figura 4. Balances de exergía del horno de la planta No.2 (antes y óptimo) y del horno de KFN.

La figura 4 muestra una mejora en la eficiencia exergética del horno. En este caso se puede apreciar una tendencia del horno hacia el comportamiento del horno de KFN.

Como se puede apreciar las irreversibilidades de la combustión (I-comb) tienden a disminuir mientras que las irreversibilidades de la transferencia de calor y momento (I-TCM) tienden a aumentar su porciento. Lo que quiere decir, que con la optimización aumenta la exergía destruida durante los procesos de transferencia de calor relacionados con la calcinación de calizas, lo que incide en el aumento de la calidad de la cal viva.

En estudios anteriores, basados en el 1er principio de la termodinámica $[14,16,17,18,19$, 20] se identificaron las pérdidas con los gases de escape como la principal ineficiencia del proceso. El análisis con la segunda ley permite identificar, además de las pérdidas con los gases de escape a las irreversibilidades de la combustión y de la transferencia de calor y momento como los factores que más inciden en las ineficiencias del proceso. Por otra parte, al considerar la influencia de la calidad de la cal sobre su precio de venta y por consiguiente sobre el margen de contribución económica de la calcinación de calizas, es posible optimizar el proceso económica (al concentrar, solo en el consumo de combustible, alrededor del $50 \%$ de los costos totales de producción [2, 3]), energética (más del $90 \%$ de la energía de la producción de cal se consume en la calcinación de calizas [36]) y ambientalmente (más del $80 \%$ de los impactos ambientales de la producción de cal se concentran en la calcinación de calizas [9]) utilizando una sola función objetivo, lo que simplifica la optimización.

\section{CONCLUSIONES}

Las principales ineficiencias de la calcinación de calizas en hornos de cuba vertical se 
localizan en combustión del combustible, la transferencia de calor y momento y las pérdidas de exergía con los gases de escape, contribuyendo con más del $49 \%$ de la pérdida de eficiencia exergética.

La implementación de la metodología de optimización permitió reducir el consumo de energía en un $4,6 \%$ e incrementar la calidad de la cal en $5,3 \%$ pasando a operar con un margen de contribución económica negativo a uno positivo. Considerar la influencia de la calidad de la cal sobre el margen de contribución económica permite optimizar la calcinación de calizas en el plano económico, energético y ambiental, minimizando los parámetros a controlar.

\section{REFERENCIAS BIBLIOGRÁFICAS}

[1] Oates, J.A.H., Lime and Limestone. WileyVCH, Weinheim, Alemania, 1998.

[2] Bes A. Dynamic process simulation of limestone calcination in normal shaft kilns. Tesis doctoral. Magdeburg: Otto-von-Guericke University; 2006.

[3] Ochoa, P.A., Sagastume, A., Vandecasteele, C., 2010. Cleaner production in a small lime factory by means of process control. Journal of Cleaner Production. 18, 1171-1176.

[4] Federal German Association of the Lime Industry, 2005. Annual general meeting of the federal German association of the lime industry. ZKG International. 58, 24-25.

[5] Federal German Association of the Lime Industry, 2006. Energy prices put a strain on the lime industry. ZKG International. 59, 22-23.

[6] Benhelal, E., Zahedi, G., Hashim, H., 2012. A novel design for green and economical cement manufacturing. Journal of Cleaner Production 22, 60-66.

[7] Freiherr, D., 2004. The effects of emissions trading on the German lime industry. ZKG international. 57, 50-56.

[8] Wolter, A., Fuchs, W., 2007. Specific $\mathbf{C O}_{2}$ emissions and the applications of lime burning kilns. ZKG international. 60, 45-50.

[9] Sagastume, A., Van Caneghem, J., Cogollos, J.B., Vandecasteele, C., 2012. Evaluation of the environmental performance of lime production in Cuba. Journal of Cleaner Production 31, 126-136.

[10] Borgwardt, R.H. Calcium oxide sintering in atmospheres containing water and carbon dioxide. Ind. Eng. Chem. Res. 28 (4) 493-500, 1989.

[11] Commandre, J.M., Salvador, S., Nzihou, A., Reactivity of laboratory and industrial limes. Chemical Engineering Research and Design, 85 (4) $1-8,2007$

[12] Hogewoning, S., Wolter, A., Schmidt, S.O., Dependence of hard burn potential on limestone properties (Part 1). ZKG International 61 (6) 5460, 2008.

[13] Hogewoning, S., Wolter, A., Schmidt, S.O., Dependence of hard burn potential on limestone properties (Part 2). ZKG International 61 (7) 8493, 2008.

[14] Boynton, R.S., Chemistry and technology of lime and limestone, 2nd ed., John Wiley \& Sons, New York, 1980.

[15] European Commission, 2001. Integrated Pollution Prevention and Control (IPPC). Reference document on best available techniques in the cement and lime manufacturing industries. Disponible en: www.epa.ie/downloads/advice/brefs/cement.pdf (13.11.2012).

[16] Ruch, H., The theoretical limits of the heat consumption in lime burning considered on the basis of the physico-chemical relationships. ZKG International. 34 (1) 26, 1981.

[17] Thomas, H.P., 1981. Limits of the heat consumption of annular shaft kilns at combustion of lime. ZKG International. 34, 27-35.

[18] Schaefer, G., 1981. Heat consumption limits of parallel-flow /counter-flow at combustion of lime regenerative kilns. ZKG International. 34, 3641.

[19] Hill, N., Mason, K., 1997. Calculating the energy efficiency of a lime burning process. World Cement. 28, 52-56.

[20] Senegacnik, A., Oman, J., 2008. Improving the efficiency of lime burning annular shaft kiln by gas recirculation. Revista ciências exatas. 2, 1-10. 
[21] Egorov, A.A., Sidorov, N.V., Tsibulnikov, A.I., Zyulyarkin, D.A., Kurochkin, B.M., 1982. Increasing the thermal efficiency of lime kilns. Metallurgist. 26, 252-254.

[22] Piringer, H., Werner, W., 2008. Conversion of large-diameter single shaft kilns to lignite dust firing successfully concluded. 61, 46-52.

[23] Foster, John B., Method for the control and command of a kiln for the burning of lumped material and the relative kiln. European Patten Office. 2011.

[24] Sheng-xiang, D., Qing-song, X., Jie-min, Z., 2011. A lime shaft kiln diagnostic expert system based on holographic monitoring and real-time simulation. Expert Systems with Applications. 38, $15400-15408$.

[25] Zuidelveld, P.L., van den Berg, P.J., 1971. Design of lime shaft kilns. Chemical Engineering Science. 26, 875-883.

[26] Piringer, H., Loebner, A., 2004. Optimization of capital costs during the development of a new generation of Maerz PFR lime kilns. ZKG international. 57, 40-49.

[27] Zheng-ming, Y.I., Jie-min Z., Hong-rong, C., 2005. Numerical simulation of thermal process and energy saving of lime furnace. J. Cent. South uni. Technol. 12, 295-299.

[28] Brechet, T., Tulkens, H., 2009. Beyond BAT: Selecting optimal combinations of available techniques, with an example from the limestone industry. Journal of Environmental Management. 90, 1790-1801.

[29] Okonkwo, P.C., Adefila, S.S., 2012. The Kinetics of Calcination of High Calcium Limestone. International Journal of Engineering Science and Technology. 4, 391-400.

[30] Wolter, A., 2000. Present situation and future outlook for burning fine-grained limestone. ZKG international. 53, 518-524.

[31] Sagastume, A., Cogollos, C., Vandecasteele, C., 2013. Energy and exergy assessments of a lime shaft kiln, Applied Thermal Engineering 51, 273 280.
[32] Do, D.H., Simulation of lime calcination in normal shaft and parallel flow regenerative kilns. Magdeburg: Otto-von-Guericke University, 2012.

[33] Sagastume, A., Vandecasteele, C., 2011. Exergy-based indicators to evaluate the possibilities to reduce fuel consumption in lime production. Energy. 36, 2820-2827.

[34] Gosselin, L., Tye-Gingras, M., Mathieu-Potvin, F., 2009. Review of utilization of genetic algorithms in heat transfer problems. International Journal of Heat and Mass Transfer. 52, 2169-2188.

[35] Kellenberger, D., Althaus H. J., Jungbluth, N., Kunniger, T., Lehmann, M., Thalmann, P., Ecoinvent Report No.7, Life Cycle Inventories of Building Products. Final Report Ecoinvent Data v2.0 No.7. EMPA Dunbendorf, Swiss Centre for Life Cycle Inventories, Dubendorf, CH. 2007.

[36] Sagastume, A., Optimización de la calcinación de calizas en hornos de cuba vertical normal. Cienfuegos: Universidad de Cienfuegos, 2013. 